\section{FLT3 ligand plasma levels in acute myeloid leukemia}

This prospective single-center study was designed to assess soluble Fms-like tyrosine kinase 3 ligand concentrations (sFLc) during the treatment of acute myeloid leukemia (AML). Three different kinetic profiles were identified during induction and found to have a significant impact on outcomes, especially survival outcomes.

Fms-like tyrosine kinase 3 (FLT3) ligand (FL) is a key regulator of hematopoiesis. ${ }^{1}$ sFLc have been shown to correlate with the extent of bone marrow aplasia after radiotherapy or chemotherapy. ${ }^{2-4}$ Interestingly, FL is expressed by leukemic cells and might enhance proliferation through an autocrine process. ${ }^{5-8}$ Moreover, high soluble levels of FL may explain resistance to FLT3 inhibitors. ${ }^{9}$ In a previous phase I study, testing a radioimmunotherapy regimen for relapsed/refractory acute lymphoblastic leukemia, we observed that only the responders displayed sustained increased sFLc. ${ }^{10}$ Apart from this report, data regarding the prognostic impact of FL levels in leukemia are lacking. We therefore designed a prospective study to investigate the potential prognostic impact of the kinetic profile of sFLc on outcomes in patients with AML.

The FLAM/FLAL trial was a prospective single-center, non-interventional study conducted at Nantes University Hospital (Nantes; France). It was planned to include all consecutive adult ( $\geq 18$ years old) patients with AML (except pro-myelocytic AML) treated intensively with first-line therapy from May 2016 for an 18-month period.
Our aim was to assess the impact of sFLc kinetic profile on outcomes. Parameters considered were refractory status after induction, relapse, progression-free survival and overall survival. AML cases were treated according to standard-of-care, first-line therapy (Online Supplementary File). Younger AML patients ( $<60$ years old) with favorable features were treated according to the protocol of the LAM2006CBF trial ${ }^{11}$ while other cases were treated within the ongoing BIG study ( $w$ ww. ClinicalTrials.gov NCT02416388). All older AML patients ( $\geq 60$ years old) were treated according to the protocol of the LAMSA2002 trial. ${ }^{12}$ Some older AML patients with favorable features received intensive consolidation with intermediate-dose cytarabine. Allogeneic transplantation was performed according to the criteria of each protocol.

sFLc (expressed in $\mathrm{pg} / \mathrm{mL}$ ) was assayed from thawed plasma samples by enzyme-linked immunosorbent assay (DY308, R\&D Systems, Minneapolis, MN, USA). These plasma samples had to be collected on days 1, 8, 15 and 22 of induction. All patients provided informed consent to participation in the study. The protocol had been approved by the ethical committee of Nantes University Hospital (GNEDS; ref: RC15_0374), the French Health Ministry (ref: 16-226) and the French National Commission on Informatics and Liberty (CNIL: ref: 2001209v0). The trial was registered at www. ClinicalTrials.gov NCT02693899.

The prognostic value of sFLc and sFLc kinetic profile was assessed according to: (i) refractory status after induction $(\geq 5 \%$ bone marrow blasts or persistent aplasia $>45$ days) or (ii) relapse, whether morphological ( $\geq 5 \%$

Table 1. Characteristics of the patients studied.

\begin{tabular}{|c|c|c|c|c|c|}
\hline & All patients (N=62) & FLI group (N=26) & FLD group (N=22) & FLS group (N=14) & $P$ \\
\hline Follow-up: days (range) & $543(154-787)$ & $558(154-787)$ & $567(165-757)$ & $194(161-488)^{*}$ & \\
\hline Gender: males & $32(52 \%)$ & 10 & 10 & 12 & 0.01 \\
\hline \multicolumn{6}{|l|}{ Age } \\
\hline Median: years (range) & $59(28-71)$ & $62(28-71)$ & $58(33-69)$ & $57(36-68)$ & 0.14 \\
\hline$<60$ years, n (\%) & $33(53 \%)$ & 10 & 14 & 9 & 0.14 \\
\hline \multicolumn{6}{|l|}{ ELN 2010 classification } \\
\hline Favorable, n (\%) & $18(29 \%)$ & 6 & 9 & 3 & 0.21 \\
\hline Int $1+$ int 2, n (\%) & $32(52 \%)$ & 16 & 10 & 6 & \\
\hline Adverse, n (\%) & $12(19 \%)$ & 4 & 3 & 5 & \\
\hline \multicolumn{6}{|l|}{ WHO AML type } \\
\hline Not otherwise specified, n (\%) & $21(34 \%)$ & 7 & 8 & 6 & 0.56 \\
\hline MDS-related, n (\%) & $11(18 \%)$ & 5 & 2 & 4 & \\
\hline Recurrent cytogenetic abnormality, n (\%) & $25(40 \%)$ & 11 & 10 & 4 & \\
\hline Therapy-related, n (\%) & $5(8 \%)$ & 3 & 2 & 0 & \\
\hline Median \% of blasts at diagnosis (range) & $54(20-94.5)$ & $51(20-94.5)$ & $58(20-94)$ & $51(25-68)$ & 0.68 \\
\hline <median, n (\%) & $32(52 \%)$ & 14 & 9 & 9 & 0.37 \\
\hline \multicolumn{6}{|l|}{ White blood cell count at diagnosis } \\
\hline Median $x 10^{9} / \mathrm{L}$ (range) & $5.8(0.5-236)$ & $5.8(0.6-236)$ & $2.3(0.5-118)$ & $4.9(0.9-121)$ & 0.69 \\
\hline$<20$ x10 $/ \mathrm{L}, \mathrm{n}(\%)$ & $44(71 \%)$ & 18 & 15 & 11 & 0.77 \\
\hline \multicolumn{6}{|l|}{ Type of consolidation ${ }^{\circ}$} \\
\hline Intensive, n (\%) & $36(58 \%)$ & 14 & 15 & 7 & 0.30 \\
\hline Non-intensive, n (\%) & $18(29 \%)$ & 11 & 5 & 2 & \\
\hline Allograft, n (\%) & $40(64,5 \%)$ & 17 & 13 & 10 & 0.74 \\
\hline
\end{tabular}


bone marrow blasts after complete remission), cytogenetic (reappearance of chromosomal abnormalities), molecular (reappearance of molecular abnormalities) or immunophenotypic (reappearance of a blast population detected by flow cytometry). Parameters known to have an impact on outcomes in AML were taken into account in the univariate and/or multivariate analyses: age ( $<$ versus $\geq 60$ years old), blast percentage at diagnosis (relative to median), European LeukemiaNet (ELN) 2010 risk classification $^{13}$ and diagnostic white blood cell count ( $\leq$ versus $>20 \times 10^{9} / \mathrm{L}$ ). Quantitative variables were described by the median (range) and compared by a Wilcoxon rank-sum test. Categorical variables were described as counts and percentages and compared by Wilcoxon or Fisher exact tests when appropriate. Survival probabilities are presented as percentages with $95 \%$ confidence intervals $(95 \% \mathrm{CI})$. For univariate analyses, progression-free survival and overall survival were estimated by log-rank tests and Kaplan-Meier graphs. Multivariate analyses were performed using the Cox proportionalhazard model. Factors differing between the two groups in terms of distribution and factors significantly associated with outcome were included in the multivariate analysis. Hazard ratios and cause-specific hazard ratios are given with $95 \%$ confidence intervals. All tests were twosided and $P$ values $<0.05$ were considered statistically significant. Statistical analyses were performed in June 2018 using the $\mathrm{R}$ and Medcalc (Ostend, Belguim) software packages.

Between May 2016 and January 2018, 63 AML patients were included in the study. sFLc were ultimately available for 62 patients. Their median age was 59 years (range, 29-71) and median follow-up for alive patients was 541 days (range, 154-787). The patients' characteristics are shown in Table 1 . A total of 242 samples were analyzed during induction. The median sFLc at day 1

sFLC blastic profile of the three groups

FLI: Sustained increase of sFLc from day 1 to day 22 of induction $(n=26)$.

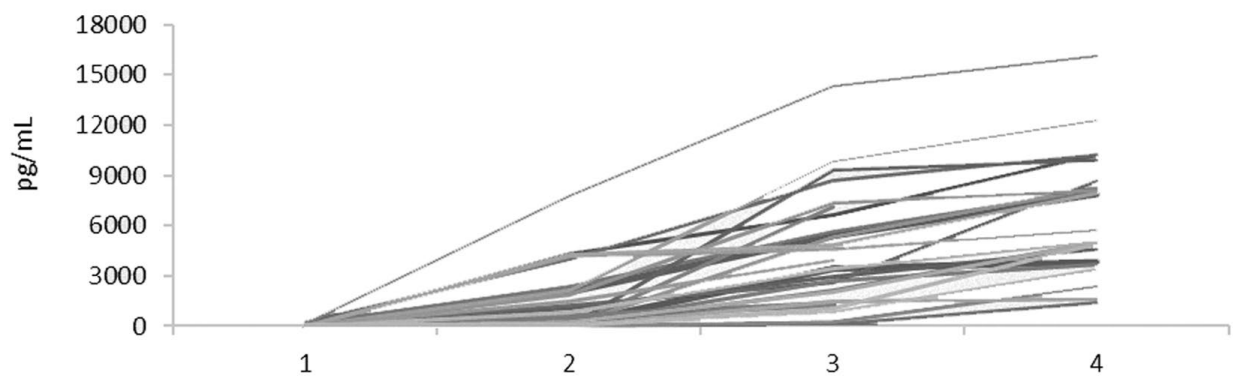

FLD: Increase of sFLc between days 1 and 15 , then decrease until day 22 of induction $(n=22)$.

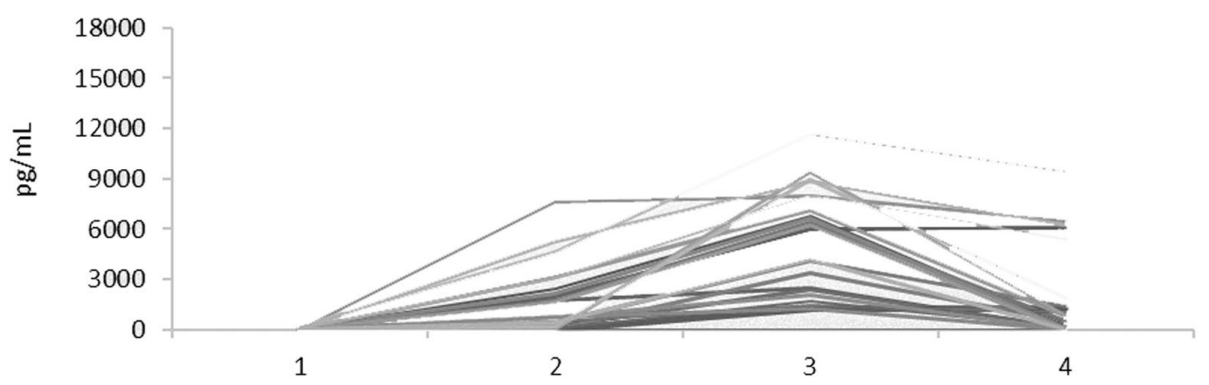

FLL: Stable low levels of sFLc $(<1000 \mathrm{pg} / \mathrm{mL})$ between day 1 and day 22 of induction $(\mathrm{n}=14)$.

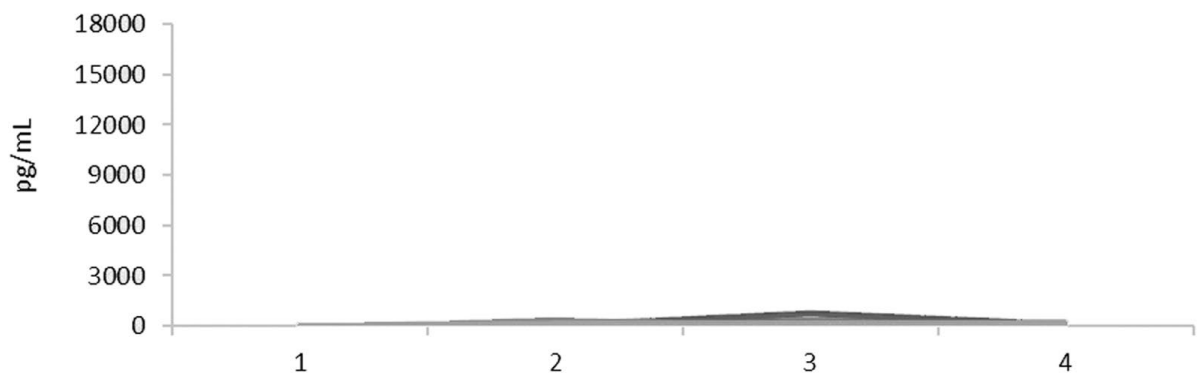

Figure 1. Three different kinetic profiles of soluble FLT3 ligand concentrations during induction treatment in patients with acute myeloid leukemia. FLI: sustained increase of soluble FLT3 ligand concentrations (sFLc) from day 1 to day 22 of induction ( $n=26)$. FLD: an increase of sFLc between days 1 and 15 , followed by a decrease until day 22 of induction $(n=22)$. FLL: stable, low sFLc $(<1000 \mathrm{pg} / \mathrm{mL})$ between day 1 and day 22 of induction $(n=14)$. X axis: $1=$ day $1 ; 2=$ day $8 ; 3$ = day $15 ; 4$ = day 22 . 
$(n=62)$, day $8(n=62)$, day $15(n=59)$ and day $22(n=59)$ were 2 (range, 0-234), 321 (range, 0-7750), 2952 (range, $0-14284$ ) and 1390 (range: $13-16088$ ) pg/mL, respectively. Analysis of the results revealed three sFLc kinetic profiles: (i) a sustained increase from days 1 to 22 (FLI group), (ii) an increase from days 1 to 15 , then a decrease at day 22 (FLD group) and (iii) stable, low levels $(<1000$ $\mathrm{pg} / \mathrm{mL}$ ) throughout, from days 1 to 22 (FLL group) (Figure 1). Twenty-six patients (42\%) were classified as FLI, 22 $(35 \%)$ as FLD and $14(23 \%)$ as FLL. Three patients who achieved neutrophil recovery before day 22 with a sustained sFLc increase from days 1 to 15 were classified as FLI. Two patients who displayed a sustained sFLc increase from days 1 to $15(>1000 \mathrm{ng} / \mathrm{mL})$, then stable sFLc were classified as FLD. Median sFLc at days 1, 8, 15 and 22 were as follows for the three groups: FLI: 2 (range, 0-234), 724 (range, 0-7750), 3673 (range, 65-14284) and 5753 (range, 1390-16088) pg/mL; FLD: 6 (range, 0-177), 1229 (range, 4-7666), 6019 (range, 1217-11640) and 684 (range, 14-9428) pg/mL; and FLL: 0 (range, 0-34), 60 (range, 0-419), 124 (range, 0-800) and 81 (range, 13-213) $\mathrm{pg} / \mathrm{mL}$. Although median sFLc were similar on day 1 $(P=0.18)$, significant differences were observed between the three groups at days $8(P=0.001), 15(P<0.001)$ and 22 $(P<0.001)$. Of note, median sFLc were significantly different between the FLI and FLD groups only at day 22 $(P<0.001)$.

There were no significant differences between the three groups regarding median age, ELN 2010 risk stratification, ${ }^{13}$ World Health Organization classification, ${ }^{14}$ median white blood cell count and bone marrow blast percentages at diagnosis. There were also no differences in terms of intensive consolidation or allografts received between the three groups (Table 1).

When comparing the three groups with different FL kinetic profiles, nearly all refractory patients $(n=6)$ belonged to the FLL group (FLL $n=5$, FLD $n=1$, FLI $n=0$; $P=0.0007)$. Seven patients out of 14 relapsed in the FLL group (morphological relapse $n=4$, molecular relapse $n=2$, immunophenotypic relapse $n=1$ ), seven out of 22 in the FLD group (morphological relapse $n=4$, molecular relapse $n=2$, immunophenotypic relapse $n=1$ ) and three out of 26 in the FLI group (all morphological relapses). The inci- dence of relapse was significantly higher in the FLL group $(P=0.0009)$.

In univariate analyses (Online Supplementary File), the 2year progression-free and overall survival rates were significantly better for the FLI group $(79.1 \% \pm 8 \%$ versus FLD $54.9 \% \pm 11 \%$ versus FLS $11.4 \% \pm 10 \% ; P<0001$; and $80.4 \% \pm 8 \%$ versus FLD $58.6 \% \pm 11 \%$ versus FLL $18.6 \%$ $\pm 10 \%, P=0.09$, respectively) (Figure 2). There was a trend for an association between 2-year progression-free survival (but not overall survival) and ELN 2010 risk stratification (favorable: $70.9 \% \pm 11 \%$, versus intermediate$1+$ intermediate- $2: 57.1 \% \pm 10 \%$ versus unfavorable $33 \%$ $\pm 13 \% ; P=0.06)$. Stratification of the patients according to the median sFLc level at day $+15(2952 \mathrm{pg} / \mathrm{mL})$ also showed significantly different 2-year progression-free survival rates, which were $38.2 \% \pm 9 \%$ for levels below the median versus $71.8 \% \pm 8 \%$ for levels above the median $(P=0.02)$. The same was true for day +22 median sFLc $(1390 \mathrm{pg} / \mathrm{mL})$ with the progression-free survival rates being $38.9 \% \pm 9 \%$ versus $73.6 \% \pm 8 \%(P=0.02)$ for patients with levels above and below the median, respectively. Age did not have an impact on either progressionfree survival or overall survival.

Multivariate analysis (Online Supplementary File) considering age, ELN stratification, day 15 and day 22 sFLc showed that the sFLc kinetic profile remained the most powerful factor independently associated with progression-free survival (hazard ratio, 3.62; 95\% CI: 1.65-7.94, $P=0.001)$. In our population and with our modeling, the sFLc kinetic profile was the sole factor independently associated with overall survival (hazard ratio, 2.60; 95\% CI: $1.12-6,07, P=0.02$ ).

We identified three sFLc kinetic profiles during AML induction and found that these had a strong and significant impact on both progression-free survival and overall survival. Indeed, stable low levels of sFLc throughout induction (FLL profile) appear to predict not only a poor response but also a high incidence of relapse and very poor survival outcomes. In contrast, patients with a sustained increase in sFLc from day 1 to day 22 (FLI profile) showed better outcomes with a very low incidence of relapse. The FLD profile with a non-sustained increase of sFLc was associated with an intermediate prognosis. This

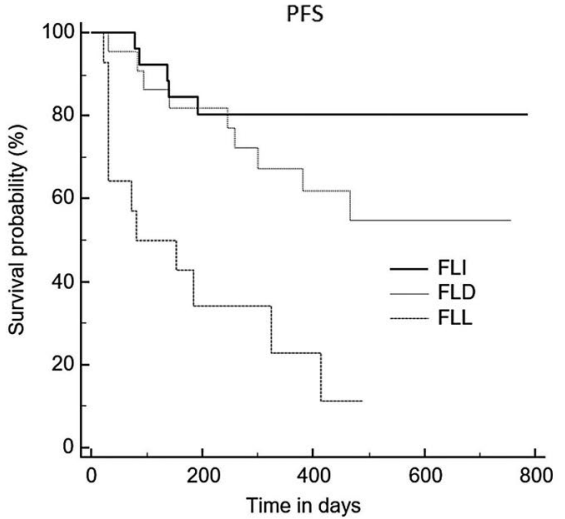

$P<0.0001$
OS

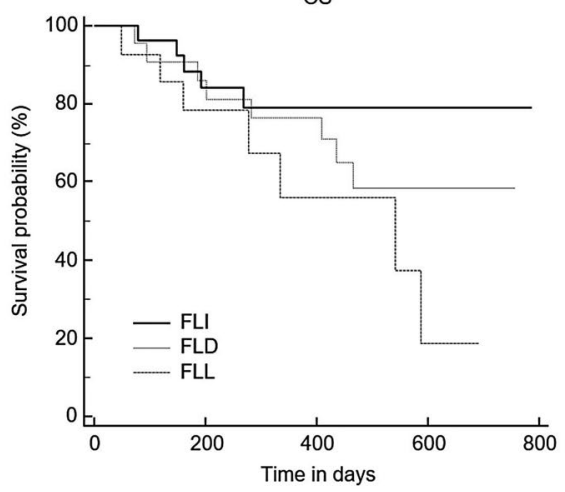

Figure 2. Progression-free and overall survival rates according to the three kinetic profiles of soluble FLT3 ligand concentrations during induction treatment in patients with acute myeloid leukemia. PFS: progression-free survival; OS: overall survival; FLI: sustained increase of soluble FLT3 ligand concentrations (sFLc) from day 1 to day 22 of induction; FLD: an increase of sFLc between days 1 and 15, followed by a decrease until day 22 of induction; FLL: stable, low sFLc throughout induction. 
new prognostication was able to predict outcomes better than the ELN2010 risk stratification could..$^{13}$ This may be due to the fact that ELN predictors were taken into account in the management of AML patients (i.e. improving the outcome of patients with adverse criteria through adapted treatment) and thus strengthens the value of sFLc as an independent parameter.

In this study the sFLc kinetic profiles during induction had a strong prognostic impact. One hypothesis to explain our observation is that a sustained increase of sFLc during induction reflects leukemic blast lysis and release of soluble FL into the serum/plasma. Indeed, FL is known to be expressed by leukemic cells and to stimulate the FLT3 receptor via an autocrine process that promotes leukemic cell proliferation. ${ }^{5-8}$ This hypothesis could be supported by assessing soluble FLT3 receptor during blast lysis, which does not seem to have been done so far. Another hypothesis is that persistent leukemia suppresses the bone marrow microenvironment from producing $\mathrm{FL}$ and/or over regenerative cytokines.

In conclusion, the sFLc kinetic profile during induction appears to be a powerful early prognostic marker to take into account as it may help to classify AML patients better. These results need to be validated in a larger cohort of AML patients.

Pierre Peterlin, ${ }^{1,2}$ Joelle Gaschet, ${ }^{2}$ Thierry Guillaume, ${ }^{1,2}$ Alice Garnier, ${ }^{1}$ Marion Eveillard, ${ }^{2,3}$ Amandine Le Bourgeois, ${ }^{1}$ Michel Cherel, ${ }^{2,4}$ Camille Debord, ${ }^{3}$ Yannick Le Bris, ${ }^{2,3}$ Olivier Theisen, Béatrice Mahé,, Viviane Dubruille, ${ }^{3}$ Catherine Godon, ${ }^{3}$ Nelly Robillard, ${ }^{3}$ Soraya Wuilleme, ${ }^{3}$ Cyrille Touzeau, ${ }^{1}$ Thomas Gastinne, ${ }^{1}$ Nicolas Blin, ${ }^{1}$ Anne Lok, 'Antoine Bonnet, Steven Le Gouill, 1,2 Philippe Moreau, ${ }^{1,2}$ Marie-C Béné, ${ }^{2,3}$ and Patrice Chevallier, ${ }^{1,2}$

${ }^{1}$ Hematology Clinic, CHU Nantes; ${ }^{2}$ CRCINA, INSERM, CNRS, Université d'Angers, Université de Nantes; ${ }^{3}$ Hematology Biology, CHU, Nantes and ${ }^{4}$ Nuclear Medicine Unit, ICO Cancer Center Gauducheau, Saint Herblain, France

Acknowledgments: we thank Juliette Brouazin for data management, Pauline Bargain, Lina Benaniba and Sebastien Gouard for their technical expertise with the sFLc ELISA assay, and the biological resource centre for biobanking [(CHU Nantes, Hôtel Dieu, Centre de Ressources Biologiques, Nantes, France (BRIF : BB-0033-00040)].

Funding: this study was supported by a grant from the $D H U$ Oncogreffe of Nantes.
Correspondence: PIERRE PETERLIN.

pierre.peterlin@chu-nantes.fr

doi:10.3324/haematol.2018.209460

Information on authorship, contributions, and financial \& other disclosures was provided by the authors and is available with the online version of this article at www. haematologica.org.

\section{References}

1. Tsapogas P, Mooney CJ, Brown G, Rolink A. The cytokine Flt3-ligand in normal and malignant hematopoiesis. Int J Mol Sci. 2017;18(6).pii:E1115.

2. Bertho JM, Demarquay C, Frick J, et al. Level of Flt3-ligand in plasma: a possible new bio-indicator for radiation-induced aplasia. Int J Radiat Biol. 2001;77(6):703-712.

3. Prat M, Frick J, Laporte J-P, Thierry D, Gorin N-C, Bertho J-M. Kinetics of plasma FLT3 ligand concentration in hematopoietic stem cell transplanted patients. Leuk Lymphoma. 2006:47(1):77-80.

4. Haidar JH, Bazarbachi A, Mahfouz R, Haidar HA, Jaafar H, Daher R. Serum Flt3 ligand variation as a predictive indicator of hematopoietic stem cell mobilization. J Hematother Stem Cell Res. 2002;11(3):533538.

5. Meierhoff G, Dehmel U, Gruss HI, et al. Expression of FLT3 receptor and FLT3-ligand in human leukemia-lymphoma cell lines. Leukemia. 1995;9(8):1368-1372.

6. Brasel K, Escobar S, Anderberg R, de Vries P, Gruss HJ, Lyman SD. Expression of the flt3 receptor and its ligand on hematopoietic cells. Leukemia. 1995;9(7):1212-1218.

7. Drexler HG. Expression of FLT3 receptor and response to FLT3 ligand by leukemic cells. Leukemia. 1996;10(4):588-599.

8. Zheng R, Levis M, Piloto O, et al. FLT3 ligand causes autocrine signaling in acute myeloid leukemia cells. Blood. 2004;103(1):267-274.

9. Sato T, Yang X, Knapper S, et al. FLT3 ligand impedes the efficacy of FLT3 inhibitors in vitro and in vivo. Blood. 2011;117(12):3286-3293.

10. Chevallier P, Eugene T, Robillard N, et al. (90)Y-labelled anti-CD22 epratuzumab tetraxetan in adults with refractory or relapsed CD22positive B-cell acute lymphoblastic leukaemia: a phase 1 dose-escalation study. Lancet Haematol. 2015;2(3):e108-117.

11. Jourdan E, Boissel N, Chevret S, et al. Prospective evaluation of gene mutations and minimal residual disease in patients with core binding factor acute myeloid leukemia. Blood. 2013;121(12):2213-2223.

12. Pigneux A, Béné MC, Guardiola $\mathrm{P}$, et al. Addition of androgens improves survival in elderly patients with acute myeloid leukemia: a GOELAMS Study. J Clin Oncol. 2017;35(4):387-393.

13. Döhner H, Estey EH, Amadori S, et al. Diagnosis and management of acute myeloid leukemia in adults: recommendations from an international expert panel, on behalf of the European LeukemiaNet. Blood. 2010;115(3):453-474.

14. Arber DA, Orazi A, Hasserjian R, et al. The 2016 revision to the World Health Organization classification of myeloid neoplasms and acute leukemia. Blood. 2016;127(20):2391-2405. 